\title{
Der Vorsorge-Wahnsinn: Gesund zum Arzt, krank nach Haus
}

Sicosing ie suchen eine unabhängige objektive Information über ihren Arzt? Machen Sie Gebrauch von der Zwangsabgabe der öffentlich rechtlichen Rundfunkanstalten und enthirnen Sie sich in einer illustren Talkrunde, zum Beispiel jener von Maybrit Illner. Zum Gespräch geladen sind: ein Hausarzt, ein Statistiker, ein Krebsforscher, ein Kassenvorstand und eine Staatssekretärin aus dem Gesundheitsministerium. Im Visier: IGeL-Leistungen. Sämtliche vom Arzt angebotenen Leistungen, welche die GKV nicht bezahlt, seien eine Patientenabzocke - von der Ultraschall-, Eigenblut-, Sauerstoff- und Stoßwellentherapie bis zur Krebsfrüherkennungsmaßnahme. Letztere ist besonders schädlich, da die Ergebnisse aufgrund nicht valider Datenlage dem Patienten unnötig ein Krebsrisiko suggerieren. Die nachteiligen Konsequenzen: psychische Belastung oder gar unnötige Therapien.

$\mathrm{Zu}$ IGeL-Leistungen befragt, läuft der Hausarzt in der Rolle des Anwalts der Verfemten und Geknechteten zur Hochform auf. In gestelzten Sätzen erklärt er die Verwerflichkeit dieser reinen Patientenabzocke. Er huldige nur der evidence based medicine, sei damit voll ausgelastet und käme in der Gemeinschaftspraxis damit bestens zurecht. Applaus!

Die Erfahrung mit diesen geölten Technokraten lehrt, dass ihr medizinisches Talent meist den Beginn einer Behandlung markiert. Bleibt die evidenzbasierte therapeutische Wirkung aus und beharrt der Patient auf seinen Beschwerden, wird gern ein MRT veranlasst, das nicht selten weder für die Diagnostik noch als Entscheidungshilfe für eine weitere konservative Therapie taugt. Die Weste bleibt rein, denn der Patient wird zum Orthopäden überwiesen. Der kann sich nun Rat auf der HausarztHomepage holen, die brav GKV-konform auf den
IGeL-Monitor verweist. Dort kann jeder medizinische Dummkopf (MDK) lesen, was er evidenzbasiert und leitliniengerecht zu tun hat. Die Stoßwellentherapie beim Tennisellenbogen sei schädlich, bei der Kalkschulter von fraglichem Nutzen, aber nachgewiesen schädlich. Als Therapie empfohlen werden für beide Diagnosen die „reinen“ GKV-Leistungen: Physiotherapie, Schmerztabletten, Spritzen, unter Umständen Operation. Erfahrungen eines einfachen Orthopäden: Patient hat Schmerzen, der Magen auch, Budget erschöpft, Stimmung gereizt. Wer jetzt noch lacht, hat Humor!

In der Talkrunde eskaliert die Verurteilung der Vorsorgeuntersuchung, durch statistische Zahlenspielchen belegt, bis es dem Krebsforscher zu heiß wird und er eine Lanze für die Vorsorge bricht. Der Hausarzt stimmt zu, sofern er dem Patienten das Ergebnis erkläre. Und es gebe ganz vereinzelt auch sinnvolle IGeL-Leistungen. So führe er Risikoberatungen durch, wobei er das individuelle Risikoprofil eines Patienten, zum Beispiel durch Rauchen, Bluthochdruck, Hypercholesterinämie et cetera, rechnergestützt ermittle. Nun fällt dem IKK-Mann noch ein, dass er im Zwei-Jahres-Rhythmus einen Gesundheitscheck machen lasse und die Sendung ist aus.

Und mir fällt Friedrich Schlegel ein: „Was gute Gesellschaft genannt wird, ist meist nur ein Mosaik geschliffener Karikaturen.“

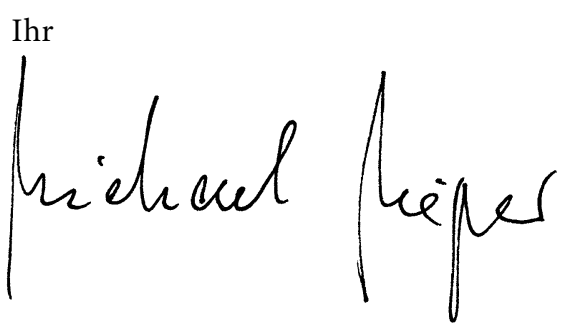

